\title{
Prevalência de Lesões Precursoras do Câncer Anal em Indivíduos HIV Positivos, Atendidos na Fundaçào de Medicina Tropical do Amazonas, Experiência Inicial em Manaus
}

\author{
Prevalence of Precursory Lesions of Cancer in HIV positive, Individuals Assisted \\ at Fundação de Medicina Tropical do Amazonas, Initial Experience in Manaus
}

\author{
FELICIDAD SANTOS GIMENEZ'; IVANTRAMUJAS DACOSTAE SILVA²; ADRIANAGONÇALVES DAUMAS \\ PINHEIRO GUIMARÃES ${ }^{3}$; LUIZ CARLOS DE LIMAFERREIRA4; JOSÉ DE RIBAMAR ARAÚJO ${ }^{5}$; RENATAPINA \\ ROCHA $^{6} ;$ LARISSADE SOUZAATALA $^{7}$; SABRINA VELOSO AVI ${ }^{8} ;$ SINÉSIOTALHARI $^{9}$
}

\begin{abstract}
${ }^{1}$ Mestranda em Patologia Tropical pela Universidade Federal do Amazonas; ${ }^{2}$ Prof. Assistente do Departamento de Clínica Cirúrgica da Universidade Federal do Amazonas; ${ }^{3}$ Profa . Auxiliar I do Departamento de Clínica Cirúrgica da Universidade Federal do Amazonas; ${ }^{4}$ Prof. do Departamento de Patologia e Medicina Legal da Universidade Federal do Amazonas; ${ }^{5}$ Gerente de Anatomia Patológica da Fundação de Medicina Tropical do Amazonas; ${ }^{6}$ Acadêmica de medicina da Universidade Federal do Amazonas; ${ }^{7}$ Acadêmica de medicina da Universidade Federal do Amazonas; 8 Acadêmica de medicina da Universidade Federal do Amazonas; ${ }^{9}$ Diretor Presidente da Fundação de Medicina Tropical do Amazonas.
\end{abstract}

GIMENEZ FS; SILVA ITC; GUIMARÃES AGDP; FERREIRA LCL; ARAÚJO JR; ROCHA RP; ATALA LS; AVI SV; TALHARI S. Prevalência de Lesões Precursoras do Câncer Anal em Indivíduos HIV Positivos, Atendidos na Fundaçào de Medicina Tropical do Amazonas, Experiência Inicial em Manaus. Rev bras Coloproct, 2008;28(1): 072-076.

RESUMO: O câncer anal representa aproximadamente $2 \%$ dos cânceres colorretais. Nos últimos anos observa-se o aumento da incidência nos indivíduos HIV positivos. Este estudo teve como objetivo avaliar a prevalência de lesões intra-epiteliais escamosas anais (ASIL) em pacientes HIV+ procedentes de Manaus. Foram estudados 45 doentes HIV+ encontrando-se no exame histopatológico, os seguintes resultados: $15(35,7 \%)$ lesões de baixo grau, 3(7,1\%) lesões de alto grau e 24(57,2\%) negativos para ASIL. Houve, portanto, alta prevalência de ASIL, 42,8\%. Concluímos que a prevalência de ASIL entre os pacientes HIV positivos, da amostra estudada, é muito importante, justificando-se a implantação de um programa de acompanhamento e detecção precoce, destas lesões; pois os pacientes HIV+ representam um importante grupo de risco para o desenvolvimento do câncer anal.

Descritores: Prevalência, câncer anal, HIV.

\section{INTRODUÇÃO}

A incidência do câncer anal na população em geral é relativamente pouco freqüente, representando apenas, aproximadamente $2 \%$ de todos os cânceres colorretais ${ }^{1}$. Contudo, observa-se o aumento da incidência desse tumor em alguns grupos populacionais, denominados "de risco"; são indivíduos anorreceptivos, de ambos os sexos ${ }^{2.3}$; portadores de doenças sexual- mente transmissíveis, tais como: gonorréia, sífilis, herpes simples tipo II, infecção pelo HIV, HPV, Chlamydia trachomatis ${ }^{4,5,6}$; portadores de lesões anais crônicas que evoluam com inflamação (fístulas, fissuras, hemorróidas) ${ }^{7}$; os imunodeprimidos (transplantados) ${ }^{8}$; tabagistas $^{9}$ e doentes portadores de câncer anal relacionado a fatores genéticos ${ }^{10}$.

Dentre estes grupos, destacam-se os indivíduos infectados pelo vírus HIV, que, face à

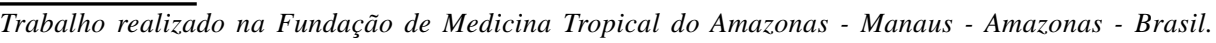

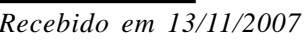

Aceito para publicação em 01/02/2008

Fonte de auxilio à pesquisa: FMT-AM

Potenciais de conflito de interesse: nenhum 
imunossupressão (menor taxa de linfócitos T CD4) ${ }^{1}$, apresentam-se mais suscetíveis aos efeitos da infecção continuada pelo HPV, estando sob risco $77 \%$ maior do que a população geral de evoluir para câncer anal. Se tiverem hábitos anorreceptivos, estes pacientes terão esta chance aumentada para $93 \%{ }^{12}$.

O comportamento do câncer anal é similar ao do câncer de colo uterino por apresentarem muitas características em comum: ambos estão associados à infecção crônica pelo vírus do papiloma humano (HPV); são precedidos por lesões precursoras não malignas, a lesão escamosa intra-epitelial cervical (CSIL) e a lesão escamosa intra-epitelial anal (ASIL); demonstram predileção pela zona de transição celular de células escamosas para glandulares; e ambos possuem características biológicas comuns, incluindo os aspectos histopatológicos. ${ }^{13}$

Diante desses aspectos, a mesma abordagem diagnóstica feita em relação às lesões precursoras do câncer cervical tem sido empregada para as lesões precursoras do câncer anal: a citologia oncótica ${ }^{14}$, a anoscopia com magnificação de imagem (AMI), e a biópsia dirigida. ${ }^{15}$

Os benefícios da detecção realizada pelo Papanicolaou cervical, com o desenvolvimento de protocolos de tratamento do câncer cervical, já foram bem comprovados: a incidência deste câncer foi reduzida de 40/100.000 mulheres para 8/100.000 mulheres nos países que adotaram esta abordagem. ${ }^{16}$

Sendo o câncer anal uma doença de instalação insidiosa, são necessários alguns anos para que uma lesão de baixo grau evolua para lesão de alto grau e desta para câncer. Portanto, se detectadas e tratadas precocemente, pode-se prevenir a transformação maligna ${ }^{17}$.

Assim, a detecção de neoplasias intra-epiteliais anais, utilizando o método do Papanicolaou-anal (Papa) e da AMI poderá identificar precocemente aqueles indivíduos sob maior risco de desenvolvimento do câncer anal e oferecer condições de abordagem terapêutica eficiente naqueles casos em que forem detectadas lesões com maiores alterações celulares ${ }^{18}$.

Apesar da alta sensibilidade (98\%), a citologia anal apresenta baixa especificidade (50\%) para prever a gravidade da lesão, daí a importância da AMI para se definir os locais onde as biópsias deverão ser feitas ${ }^{11}$. Somente o exame histopatológico poderá confirmar o grau de malignidade da lesão, já que o mesmo é considerado padrão-ouro para o diagnóstico de ASIL. ${ }^{19}$
No presente trabalho, são avaliados, através da AMI, a associação entre a presença de lesões precursoras do câncer anal e do câncer anal propriamente dito e o estado de imunodepressão de pacientes HIV+, com os objetivos de se estimar a prevalência de ASIL em grupo populacional HIV positivo de Manaus; avaliar a influência da imunodepressão e de hábitos anorreceptivos em relação a chance de apresentação de ASIL, e verificar possíveis diferenças entre os gêneros e a chance destes, anorreceptivos ou não, apresentarem ASIL.

\section{PACIENTES E MÉTODOS}

Trata-se de um estudo observacional, transversal, em que foram estudados 45 pacientes HIV+ distribuídos em 4 grupos: 6 HIV+ não anorreceptivos e não imunodeprimidos (HIV+); 7 HIV+ anorreceptivos e não imunodeprimidos (HIV+AR); $4 \mathrm{HIV}+$ não anorreceptivos imunodeprimidos (HIV+D); e $7 \mathrm{HIV}+$ anorreceptivos imunodeprimidos (HIV+ARD).

Pacientes com contagem de células CD4 $<350$ células $/ \mathrm{ml}$ e/ou carga viral acima de 10.000 cópias, foram considerados imunodeprimidos.

Todos os pacientes foram submetidos à anoscopia com magnificação de imagem e biópsia, com anestesia local, dirigida a eventuais lesões acetobrancas encontradas. Na ausência delas, biópsia de mucosa anal justapectínea proximal foram realizadas, às $7 \mathrm{~h}$. A biópsia foi encaminhada para estudo histopatológico no Laboratório de Patologia da Fundação de Medicina Tropical do Amazonas (FMT-AM). As lâminas foram coradas pela hematoxilina-eosina, e foi utilizada técnica de imunoistoquímica para a proteína p16INK4a para confirmação diagnóstica de casos duvidosos, nos quais se verificou indícios de integração do genoma do HPV de alto risco aos das células infectadas.

Os dados obtidos foram analisados sob a forma de intervalos de confiança, tabelas de contingências ou classificação cruzada e razão de chances. Para comparar a prevalência total de ASIL nos 4 grupos foi usado o teste exato de Fisher.

Significância estatística foi considerada para valores de $\mathrm{p}<0,05$.

\section{RESULTADOS}

Foram estudados 45 pacientes HIV+, sendo excluídos 3, em que o resultado do histopatológico foi insatisfatório. 
Na tabela 1 são apresentados os dados relativos à prevalência de lesões precursoras do câncer anal, segundo os grupos de pacientes estudados.

1. Grupo 1- HIV+, não anorreceptivo e não imunodeprimido.

2. Grupo 2- HIV+, anorreceptivo e não imunodeprimido.

3. Grupo 3-HIV+, não anorreceptivo e imunodeprimido.

4. Grupo 4- HIV+, anorreceptivo e imunodeprimido.

As comparações possíveis entre os grupos quanto ao risco de apresentação de ASIL, evidenciaram:

A amostra populacional estudada foi desbalanceada, pelo fato de ser composta por: $19 \%$ $\mathrm{HIV}+, 35,7 \% \mathrm{HIV}+\mathrm{AR}, 16,7 \% \mathrm{HIV}+\mathrm{D}$ e $28,6 \% \mathrm{HIV}+$ ARD. A prevalência de ASIL observada na amostra foi de $42,8 \%$ (totais nas colunas 3 e 4). O intervalo estimado com $95 \%$ de confiança para esta prevalência foi de $27,8 \%$ e $57,8 \%$. Este intervalo indica que pelo menos $27,8 \%$ dos indivíduos portadores do HIV, apresentaram alguma lesão intra-epitelial escamosa anal. A maior prevalência de lesões foi observada entre o grupo HIV+AR (19\%) (Tabela 1).

Mediante a análise dos dados da tabela 1 evidencia-se que $34,8 \%$ dos indivíduos HIV+AR e $8,7 \%$ dos indivíduos HIV+, apresentaram algum tipo de lesão intra-epitelial. A probabilidade de um indivíduo apresentar uma lesão foi de 53,3\% (8/15) para o grupo HIV+ AR e 25\% (2/8) para o grupo HIV+. A razão de chances observada foi de 3 para o grupo HIV+AR, havendo indícios de que a condição de ser HIV+AR expõe a um risco significantemente maior para o desenvolvimento de algum tipo de ASIL $(p=0,03)$.

As demais comparações entre os grupos (HIV+D versus HIV+; HIV+ARD versus HIV+; $\mathrm{HIV}+\mathrm{AR}$ versus $\mathrm{HIV}+\mathrm{ARD}$; e HIV+ARD versus HIV+D) não demonstraram diferença significativa quanto à possibilidade de um grupo estar mais propenso do que o outro quanto ao risco de apresentar ASIL.

Na tabela 2 são apresentados dados relativos à prevalência das lesões intra-epiteliais escamosas da região anal, de acordo com o gênero.

Observou-se que $14(33,3 \%)$ pacientes do sexo masculino e 4(9,5\%) dos indivíduos do sexo feminino apresentaram algum tipo ASIL. A probabilidade de um indivíduo apresentar uma lesão foi de 48,27\% (14/ 29) para o sexo masculino, e de $30,77 \%$ (4/13) para o sexo feminino, levando a uma razão de chances de 2,1 para o sexo masculino, muito embora o valor de $\mathrm{p}$ $=0,08$ indique não haver diferença significativa entre os sexos quanto à possibilidade de indivíduos HIV positivos apresentarem ou não algum tipo de ASIL (Tabela 2).

Tabela 1 - Lesões relacionadas ao câncer anal segundo os grupos de pacientes estudados.

\begin{tabular}{|c|c|c|c|c|c|}
\hline \multirow[t]{2}{*}{ Grupo de Pacientes } & \multicolumn{3}{|c|}{ Tipos de Lesões } & \multirow{2}{*}{\multicolumn{2}{|c|}{ Total }} \\
\hline & NEG & LSIL & HSIL & & \\
\hline $\mathrm{HIV+}$ & $6(14,3 \%)$ & $1(2,4 \%)$ & $1 \quad(2,4 \%)$ & 8 & $(19,0 \%)$ \\
\hline $\mathrm{HIV}+\mathrm{AR}$ & $7(16,7 \%)$ & $8(19 \%)$ & $0 \quad(0,0 \%)$ & 15 & $(35,7 \%)$ \\
\hline $\mathrm{HIV}+\mathrm{D}$ & $4 \quad(9,5 \%)$ & $2(4,8 \%)$ & $1 \quad(2,4 \%)$ & 7 & $(16,7 \%)$ \\
\hline $\mathrm{HIV}+\mathrm{ARD}$ & $7(16,7 \%)$ & $4 \quad(9,5 \%)$ & $1 \quad(2,4 \%)$ & 12 & $(28,6 \%)$ \\
\hline Total & $24(57,2 \%)$ & $15(35,7 \%)$ & $3(7,1 \%)$ & $42 *$ & \\
\hline
\end{tabular}

* 3 dados foram considerados insatisfatórios. HIV+ = Portador do vírus da imunodeficiência humana; AR = Anorreceptivo; D= Imunodeprimido; $A R D=$ Anorreceptivo e imunodeprimido; $N E G=$ negativo; $L S I L=$ Lesão intra-epitelial escamosa anal de baixo grau; HSIL Lesão intra-epitelial escamosa anal de baixo grau.

Comparação entre os grupos da tabela acima

$H I V+A R$ versus $H I V+\quad O R=3 ; p=0,03$

$H I V+D$ versus $H I V+\quad O R=2,25 ; p=0,10$

$H I V+A R D$ versus $H I V+\quad O R=2,14 ; p=0,1056$

$H I V+A R$ versus $H I V+A R D \quad O R=1,6 ; p=0,161$

$H I V+A R D$ versus $H I V+D \quad O R=1 ; p=0,7034$

$O R=$ Razão de chances 
Tabela 2 - Lesões precursoras do câncer anal em pacientes HIV+, de acordo com o gênero.

\begin{tabular}{|c|c|c|c|c|c|}
\hline \multirow[t]{2}{*}{$\overline{\operatorname{Sexo}}$} & \multicolumn{3}{|c|}{ Presença de lesões (ASIL) } & \multirow{2}{*}{\multicolumn{2}{|c|}{ Total }} \\
\hline & $\operatorname{Sim}(\%)$ & & งão (\%) & & \\
\hline Masculino & $14 \quad(33,3 \%)$ & 15 & $(35,7 \%)$ & 29 & $(69 \%)$ \\
\hline Feminino & $(9,5 \%)$ & 9 & $(21,5 \%)$ & 13 & $(31 \%)$ \\
\hline Total & $18 \quad(42,9 \%)$ & 24 & $(57,2 \%)$ & 42 & \\
\hline
\end{tabular}

Teste exato de Fisher: Valor $p=0,08$.

\section{DISCUSSÃO}

Dentre os 42 indivíduos portadores de HIV, do presente estudo, atendidos na Fundação de Medicina Tropical do Amazonas, 18 apresentaram lesões precursoras do câncer anal, conferindo uma prevalência de ASIL de 42,8\%; estando dentro da média da literatura pesquisada, onde a incidência de ASIL oscila de $36 \%^{20}$ até $78 \%{ }^{21}$.

As lesões intra-epiteliais anais mais freqüentes foram as de baixo grau (LSIL), encontradas em $15(35,7 \%)$ casos, na literatura, verifica-se grande diferença de incidência, desde $62 \%{ }^{12}, 47 \%,{ }^{11}$ até $27 \%^{15}$. O mesmo ocorre com as lesões de alto grau (HSIL) que encontramos em $3(7,1 \%)$ pacientes, e em outros trabalhos observamos valores de $54 \%^{11,15}$ até $20 \%{ }^{12}$.

O intercurso sexual anal, avaliado isoladamente, influenciou de forma substancial para o desenvolvimento de ASIL na amostra estudada, com valor $p=$ 0,03 , havendo concordância com a literatura. ${ }^{21}$ Entretanto a imunodepressão, avaliada isoladamente, não pôde ser considerada fator de risco relevante, valor $\mathrm{p}$ $=0,10$, como seria esperado que pacientes HIV+ imunodeprimidos tivessem uma incidência maior que os somente HIV+, e uma maior alteração neoplásica. ${ }^{22}$

Entre os pacientes examinados, 14 (43,75\%) eram homens e $4(30,8 \%)$ eram mulheres, os quais, foram diagnosticados com ASIL. O valor $\mathrm{p}=0,08$, Demonstrou fraca evidência de que a prevalência de lesões intraepiteliais escamosas anais esteja relacionada ao gênero.

É importante lembrar que, pelo fato da amostra estudada ser pequena e por não ter sido possível a realização do teste-reteste interobservador, os resultados aqui encontrados, devem ser considerados somente para a amostra em questão, sendo necessários maiores estudos a fim de comprová-los.

\section{CONCLUSÕES}

$\mathrm{Na}$ amostra populacional estudada, a prevalência de ASIL encontrada foi de $42 \%$. Não houve influência do fator imunodepressão, nem diferença entre os gêneros na chance de pacientes HIV positivos desenvolverem ASIL. O relacionamento sexual anorreceptivo exerceu influência substancial na chance dos pacientes estudados, apresentarem ASIL.

\footnotetext{
ABSTRACT: The anal cancer accounts approximately for $2 \%$ of the cases of colorectal cancer. In the last few years it has been observed an increase in the incidence of this disease in HIV-positive individuals. The objective of this study is to evaluate the prevalence of anal squamous intraepithelial lesions (ASIL) in HIV-positive patients which are resident in Manaus. Forty-five HIV+ patients were included in the study, from which it was collected anal mucosal tissue for biopsy under anoscopic control using widened image for histopathological exam. According to this exam, it was found the following results: $15(35,7 \%)$ low grade lesions, 3(7,1\%) high grade lesions and $24(57,2 \%)$ negative for ASIL. Therefore, there was a high prevalence of ASIL, 42,8\%. We concluded that the prevalence of ASIL among HIV-positive patients, in the studied sample, is very important, justifying the implementation of a program of precocious monitoring and detection of these lesions, as the HIV+ patients represent an important risk group for the developing of anal cancer.
} 


\section{REFERÊNCIAS}

1. Keighley MRB; Williams NS. Cirurgia do ânus, reto e colo. $1^{2}$ edição. Volume I. São Paulo: Editora Manole; 1998.

2. Daling JR; Weiss NS; Klopfenstein LL; Cochran LE; Chow WH; Daifuku R. Correlates of homosexual behavior and the incidence of anal cancer. JAMA. 1982; p 1988-90.

3. Daling JR; Weiss NS; Hislop TG; Maden C; Coates RL; Sherman KJ et al. Sexual practices, sexually transmitted diseases, and the incidence of anal cancer. N. Engl. J. Med. 1987; p 973-7.

4. Martin F; Bower M. Anal intraepithelial neoplasia in HIV positive people. Sex. Transm. Infect. 2001; p. 327-31,

5. Martins CR. HPV-induced anal displasya: what do we know and what can we do about it? Hopkins HIV Rep. 13 (3): 2001; p 3-5.

6. Chin-Hong PV; Palefsky JM. Natural history and clinical management of anal human papillomavirus disease in men and women infected with human immunodeficiency virus. Clin. Infect. Dis. 2002; p 1127-34.

7. Prieto R; Vazquez M. Anal epidermoid carcinoma: a rare incidence or a rare diagnosis? Rev Esp Enferm Dig. 1997; 89(2): p 128-32.

8. Penn I. Occurrence of cancers in immunosuppressed organ transplant recipients. Clin Transpl. 1998; p 147-58.

9. Frisch M; Glimelius B; Wohlfahrt J; Adami Ho; Melbye M. Tobacco smoking as a risk factor in anal carcinoma: an antiestrogenic mechanism? J. Natl Cancer Inst. 1999; p. 70815.

10. Frisch M; Olsen Jh; Bautz A; Melbye M. Benign anal lesions and the risk of anal cancer. N. Engl. J. Med. 1994; p 300-2.

11. Pikkety C; Darragh TM; Da Costa M; Bruneval P; Heard I; Kazatchkine MD; Palefsky JM. High prevalence of anal human papillomavirus infection and anal cancer precursors among HIV-infected persons in the absence of anal intercourse. Ann. Intern. Med. 2003; p 453-9.

12. Palefsky JM; Holly EA; Ralston ML; Jay N. Prevalence and risk factors for human papillomavirus infection of the anal canal in human immunodeficiency virus (HIV)-positive and high-risk HIV-negative homosexual men. J. Infect. Dis. 1998; p 361-7.

13. Melbye M; Sprogel P. Aetiological parallel between anal cancer and cervical cancer. Lancet,. Sep 1991 v.338, n.8768, p 657-9.
14. Costa e Silva IT; Gimenez FS; Guimarães RAG; Camelo RT; Melo MND; Barros FS; Daumas A; Cabral CRB; Guimarães EL. Citologia anal como método de rastreamento para a detecção precoce do câncer anal: esfregaços com algodão hidrófilo são mesmo insatisfatórios? Acta Cir. Bras. [serial on line] 2005; Jan-Fev, 20 (1).

15. Mathews WC; Sitapati A; Caperna JC; Barber RE; Tugend A; Go U. Measurement Characteristics of Anal Cytology, Histopathology, and High-Resolution Anoscopic Visual Impression in an Anal Dysplasia Screening Program. J. Acquir Immune Defic Syndr. 2004; 37: 1610-15.

16. Qualters JR; Lee NC; Smith RA; Aubert RE. Breast and cervical cancer surveillance, United States, 1973-1987. MMWR CDC Surveill Summ 1992; 41: p 1-7.

17. Palefsky JM; Holly EA; Ralston ML; Da Costa MM; Bonner $\mathrm{H}$; Jay $\mathrm{N}$ et al. The effect of highly active antiretroviral therapy on the natural history of anal squamous intraepithelial lesions and anal human papillomavirus infection. J Acquir Immun Defic Syndr 2001; 28: p 422-428.

18. Nadal RS, Manzione CR. Citologia Como Método Para Detecção de Lesões Precursoras do Carcinoma Anal. Rev. Bras. Coloproct., 2005; 25(1); 72-4.

19. De Ruiter A, Carter P, Katz DR, Kocjan G, Whatrup C, Northover $\mathrm{J}$, et al. A comparison between cytology and histology to detect anal intraepithelial neoplasia. Genitorin Med. 1994; 70: 22-5.

20. Palefsky JM, Holly EA, Ralston ML, Arthur SP, Jay N, Berry $\mathrm{JM}$ et al. Anal squamous intraepithelial lesions in HIV-positive and HIV-negative homosexual and bisexual men: prevalence and risk factors. J Acquir Defic Syndr Hum Retrovirol. 1998 Apr 1; 17(4): 320-6.

21. Fox PA. Human papillomavírus and anal intraepithelial neoplasia. Curr Opin Infect Dis. 2006; 19 (1): p 62-6.

22. Scholefield JH, Castle MT, Watson NF. Malignant transformation of high-grade anal intraepithelial neoplasia. $\mathrm{Br}$ J Surg. 2005; 92 (9);1133-6.

\section{Endereço para correspondência:} FELICIDAD SANTOS GIMENEZ

Avenida Rio Negro, 349 - Santo Agostinho

69036-720 - Manaus-AM

Fax: 9232323411

felicidadgimenez@yahoo.com.br 